Arias, J.; Cano, V. (2018). Contabilidad y modos de producción. Apuntes reflexivos para repensar el papel social de la contabilidad. Contaduría Universidad de Antioquia, 73, 33-48.

Doi: https://doi.org/10.17533/udea.rc.n73a02

\title{
Contabilidad y modos de producción. Apuntes reflexivos para repensar el papel social de la contabilidad ${ }^{*}$
}

\author{
Juan David Arias Suárez \\ jd.arias@hotmail.com - jdarias@poligran.edu.co \\ Institución Universitaria Politécnico Grancolombiano \\ Vanessa Cano Mejía \\ vanessacanomejia@gmail.com - vcano@correo.iue.edu.co \\ Institución Universitaria de Envigado
}

* Una primera versión de este trabajo fue presentada en el III Congreso Latinoamericano de Investigadores Contables, Medellín. Del 19 al 21 de abril de 2018. 
Contabilidad y modos de producción. Apuntes reflexivos para repensar el papel social de la contabilidad. Resumen: Este texto esboza algunas reflexiones y análisis hermenéuticos en torno al papel social de la contabilidad en los diferentes modos de producción socio-históricos y las diversas formas de desarrollo económico-productivos existentes, que emergen de los sistemas de organización sociopolítica basados en los factores económicos de producción. La visión de la contabilidad trabajada se inscribe en la vertiente interpretativa de las corrientes heterodoxas de investigación. En breves líneas, se abordan rasgos básicos de la contabilidad en el comunitarismo primitivo, el esclavismo, el feudalismo, el capitalismo mercantil, industrial, financiero y bursátil, el socialismo y el comunismo, como modos de producción que permiten repensar el papel social de la contabilidad y sus determinantes económicos para representar el rumbo colectivo de las comunidades. Las reflexiones permiten identificar relaciones históricas, perspectivas epistemológicas y características propias de la contabilidad para dar cuenta de la realidad.

Palabras clave: Contabilidad, modos de producción, organización social, investigación interpretativa, teoría e historia contable.

Accounting and production modes. Reflective remarks to rethink the social role of accounting.

Abstract: This text outlines some reflections and hermeneutical analyses around the social role of accounting in the different socio-historical production modes and the existing diverse forms of economic and productive development, emerging from the sociopolitical organization systems based on the economic factors of production. The accounting vision developed in this work is inscribed under the interpretive branch of the heterodox research trends. In brief, the study addresses basic accounting features in primitive communitarianism, slavery, feudalism, mercantilist, industrial, financial and stock-exchange capitalism, socialism and communism, as production modes enabling to rethink the social role of accounting and its economic determinants to represent the collective path of communities. The reflections allow to identify historical relations, epistemological perspectives and accounting's own characteristics to account for reality. Keywords: Accounting, production modes, social organization, interpretive research, accounting theory and history.

Comptabilité et modes de production. Des notes réfléchies pour penser au rôle social de la comptabilité Résumé: Ce texte esquisse quelques réflexions et analyses herméneutiques autour du rôle social joué par la comptabilité dans les différents modes de production socio-historiques, ainsi que les multiples formes de développement économique-productifs qui existent, émergeant des systèmes d'organisation socio-politique fondés sur les facteurs économiques de production. L'idée de comptabilité ici traitée s'inscrit dans la lignée d'interprétation des courants de recherche hétérodoxes. En quelques lignes, dans ce texte seront abordés les traits fondamentaux de la comptabilité dans le communautarisme primitif, l'esclavagisme, le féodalisme, les capitalismes mercantiliste, industriel, financier et boursier; le socialisme et le communisme en tant que modes de production permettant de repenser le rôle social de la comptabilité et ses déterminants économiques pour représenter le destin collectif des communautés. Les réflexions permettent d'identifier des rapports historiques, des perspectives épistémologiques et des caractéristiques propres à la comptabilité afin de rendre compte de la réalité.

Mots-clés: comptabilité, modes de production organisation sociale, recherche interprétative, théorie et histoire comptable

Contabilidade e Modos de Produção. Anotações reflexivas para repensar o papel social da contabilidade. Resumo: Este texto bosqueja algumas reflexões e análises hermenêuticas sobre o papel social da contabilidade nos diversos modos de produção sócio-históricos e as diferentes formas de desenvolvimento econômicoprodutivos existentes, que emergem dos sistemas de organização sociopolitica baseados nos diferentes fatores econômicos de produção. A visão da contabilidade trabalhada se encaixa no eixo interpretativo das correntes heterodoxas de pesquisa. Em poucas linhas se abordam assuntos básicos da contabilidade no comunitarismo primitivo, a escravidão, o feudalismo, o capitalismo mercantil, industrial, financeiro e da Bolsa de Valores, o socialismo e o cumunismo, como tipos de produção que permitem repensar o papel social da contabilidade e seus determinantes econômicos para representar o rumo coletivo das comunidades. As reflexões permitem identificar relações históricas, perspectivas epistemológicas e características próprias da contabilidade para encarar a realidade.

Palavras Chave: Contabilidade, modos de produção, organização social, pesquisa interpretativa, teoria e história contábil. 
Cont. udea (julio-diciembre), pp. 33-48. (c) Universidad de Antioquia-2018.

\title{
Contabilidad y modos de producción. Apuntes reflexivos para repensar el papel social de la contabilidad.
}

\author{
Juan David Arias Suárez, Vanessa Cano Mejía \\ Doi: https://doi.org/10.17533/udea.rc.n73a02
}

Primera versión recibida en julio de 2018 - Versión final aceptada en agosto de 2018

\section{Ideas contextuales}

I a contabilidad es una creación antiquísima. Su participación y actuación $L$ fáctica en la estructura social, política y económica de los seres humanos para representar las múltiples expresiones y movimientos de la riqueza ha contribuido al establecimiento de una forma de entender y relacionarse en el mundo; la riqueza, entendida en sentido amplio como una construcción social tangible o intangible con el potencial de tener características económicas, sociales, culturales y/o ambientales que pueden ser controladas o representadas de forma inteligible, es decir, susceptible del proceso contable, son transhistóricas para el papel social de este saber. La contabilidad, independientemente del hecho social o el periodo sociohistórico, ha estado presente para dar cuenta de la realidad y, a su vez, perennemente ha coadyuvado a determinar una forma de comunicación y comprensión de la vida.

Las manifestaciones de la contabilidad en la sociedad han estado presentes de forma material e inmaterial; materialmente han existido un cúmulo de expresiones sociales observables (ordenamiento de líneas y puntos, muescas y marcaciones en piedras y vasijas, inscripciones en huesos, articulaciones de cuerdas y nudos, clasificación de sistemas, tablillas, ábacos, dispositivos electrónicos, etc.) e inmaterialmente también se ha manifestado como una forma de pensamiento: una 'racionalidad controladora', desde la perspectiva del profesor Danilo Ariza (2000), y naturalizada en la humanidad por el deseo de controlar y dar cuenta de la realidad, de los sucesos del pasado, de sus fluctuaciones en el presente y de su devenir. La inteligencia humana, entendida como la capacidad de pensar-actuar con sentido, genera raciocinios 
Arias, J.; Cano, V. Contabilidad y modos de producción...

y asociaciones vinculadas a la existencia y a las riquezas, que desarrollan múltiples acciones para conocer y controlar la realidad. Si la contabilidad, puede representar y coadyuvar a controlar la riqueza de la realidad producto de ese raciocinio inteligente presente desde la existencia humana, ello se configura como una racionalidad controladora y calculante.

Quizá las manifestaciones del pensamiento contable y su racionalidad controladora en la humanidad en muchas ocasiones han sido asociadas con las formas de representación (configuración de cuentas, redes de transacciones o asociación de puntos y líneas para expresar un hecho aunado a la noción de riqueza y modernamente de propiedad), pero no en todas las condiciones sociales la contabilidad ha actuado y mediado las relaciones sociales de igual manera, ya que como lo menciona el profesor Jack Araújo, ésta responde a un contexto económico y social que moldea sus preponderancias.

De ahí que, siguiendo a Bourdieu, se pueda hablar de la contabilidad como un arbitrario histórico y cultural, es decir, como un producto netamente social y cultural, que ha tomado diferentes formas y ha respondido de manera singular, en diversas culturas y épocas de la historia, a los requerimientos heterogéneos de información y control. (Vlaemminck, 1991 citado en Cardona e Hinestroza, 2013, p. 71)

La contabilidad, por derecho propio, tiene historia en el mundo, es a su vez constructora y legado de las manifestaciones humanas. Ya lo diría el profesor Jorge Manuel Gil: "la contabilidad es uno de los ojos de nuestra cultura, y cada civilización y cada cultura determinan la manera en que el hombre observa la realidad" (2003, p. 27). Empero, una forma de entender las expresiones generadas por este saber son las múltiples representaciones materiales, pero su participación histórica y geográfica es disímil y atemporal de acuerdo con la configuración de los pueblos y la (in)evolución de las civilizaciones, por ello, la idea de modos de producción desde la economía política, como concepto aglutinante de las preponderancias en los factores económicos básicos de producción (tierra, capital y trabajo), basados en la teoría marxista, permiten configurar las relaciones histórico-económicas de acuerdo con las manifestaciones y actuaciones humanas. Esta forma de entender la sociedad permite condensar las expresiones contables por cada uno de estos modos de producción y encontrar su papel social para mediar y representar las relaciones humanas asociadas a la riqueza.

"La contabilidad, al encontrarse en tal posición, es una herramienta clave para el entendimiento general de la historia, desde arriba y desde abajo, al darnos la posibilidad de observar las dinámicas económicas, sociales y simbólicas de las diferentes sociedades" (Quinche, 2006, p. 191). Esto no quiere decir que la contabilidad sea la mejor forma de comprender o captar las manifestaciones de la realidad. La contabilidad tiene un importante grado de miopía social, pues a pesar de su constructo científico y su tecnicidad 
instrumental, su aparente 'neutralidad' y 'objetividad' están mediadas por relaciones de poder político-económico y la dimensión humana, no obstante, sigue instituyéndose como un dispositivo para representar la realidad y el contexto socioeconómico, que aunque contemporáneamente miope para abarca el plexo de dimensiones existentes, porque su corpus es muy financiero, permite ver ampliamente las dinámicas de la riqueza, cuyas formas se pueden apreciar con congruencia en las taxonomías de los modos de producción.

Este documento consta de cuatro apartados más esta breve introducción problémica; se realizan algunas precisiones acerca de los elementos fundantes del conocimiento contable para ubicar una noción amplia de la contabilidad y su función social, posteriormente se ejerce una contextualización de los modos de producción y su importancia para encontrar el papel social de la contabilidad; en un tercer momento, se presenta la relación y el papel de la contabilidad en cada modo de producción y se finaliza con algunas consideraciones interpretativas sobre el saber contable. Metodológicamente, el artículo se ubica en una fase exploratoria y descriptiva, donde se relacionan algunos hallazgos documentales que permiten caracterizar la contabilidad en cada modo de producción analizado; su desarrollo consta de un ejercicio interpretativo notaxativo de problematización del papel social de la contabilidad a través del recorrido de la categoría marxista de 'modos de producción', tomando fuentes documentales diversas para generar una dialéctica conceptual y sociohistórica de contrastación de argumentos.

\section{Conocimiento contable y su función social}

La perspectiva epistemológica y sociológica empleada en el desarrollo del presente documento se puede categorizar como un ejercicio interpretativo desde las corrientes heterodoxas de la investigación en contabilidad analizadas por la profesora Chua (1986). Ello genera una precisión de la noción de contabilidad, pues producto de las mixturas y relaciones interdisciplinares y transdisciplinares, implícitas y potenciales en el conocimiento contable (Arias y Cano, 2018), no se atiende a la visión técnico-instrumental, mecánica, cartesiana y dual de la contabilidad registral tradicional, sino que la contabilidad es vista como un campo de conocimiento disciplinar encargado de estudiar problemas socioeconómicos como el control integral de la riqueza y la representación holística de la realidad social (Sarmiento, 2007; Arias, 2017). Esta forma de entender la contabilidad se alinea con la macro-perspectiva de los modos de producción, ya que en cada uno de los periodos históricos se puede apreciar una preocupación por controlar lo económicamente existente tangible o intangiblemente (riqueza) y por representar a través de algún artefacto físico o asociación mental esa realidad. 
Arias, J.; Cano, V. Contabilidad y modos de producción...

No obstante, es de conocimiento común que la contabilidad tradicional es socialmente entendida como un instrumento neutral, totalmente racionalizado e imparcial, empero, en la práctica no es así, ya que bajo el eufemismo de "neutralidad" la contabilidad tiene el potencial disfrazar y/o construir una interpretación de la realidad económica y social contextual y legitimar transacciones de los dueños del capital a través de la preponderancia de alguna perspectiva, método, escuela contable o de cambios en los criterios de reconocimiento, medición, valoración, consolidación, control, revelación o representación contables, y que terminan estableciendo negocios, influyendo en los préstamos bancarios, determinando los tributos para el Estado o legitimando acciones interesadas de actores o colectivos específicos.

Max Weber (2010) planteó que una de las premisas básicas para la existencia del capitalismo moderno es la contabilidad racional como parámetro normativo para todas las organizaciones lucrativas que se ocupan de la satisfacción de las necesidades cotidianas, lo cual presenta una visión de la contabilidad como práctica registral tradicional y como fiel reproductora de las lógicas financieras de enriquecimiento e infinito crecimiento económicos. (Arias, 2014)

En esta vía, también se ha manifestado una visión de la contabilidad como lenguaje; más allá de la teorización de Belkaoui (1993), la contabilidad es una forma de historia per se, pues genera asociaciones de los hechos sociales con artefactos de uso común (instrumentos) o representaciones metódicas comprensibles (cuentas y causaciones), lo cual construye formas de entendimiento y comunicación, ya que logra transmitir un mensaje. La contabilidad actúa como transmisora de mensajes, sin embargo, la profundidad, el sentido y la representación social del mensaje dependerá de las relaciones de su contexto, articuladas a los imperativos económicos presentes en cada modo de producción.

Por ello es importante entender que la contabilidad está presente y coconstruye un sistema de rendición de cuentas más allá de la obligación jurídica de los funcionarios públicos por revelar la distribución y ejecución de los recursos del fisco, sino como una especie de accountability sociopolítico, 1 donde a partir de la consolidación de hechos socioeconómicos en cuentas, balances y revelaciones, la sociedad tiene acceso a una lectura de su interacción económica entorno a la riqueza y ve reflejado/representado el actuar humano en las diferentes etapas históricas. Así, la contabilidad se constituye como una relatora de la dinámica social a partir de representaciones.

1 La noción de accountability es entendida como la tradicional 'rendición de cuentas', que en un entorno no-empresarial, socialmente versa como actuación sociopolítica. 


\section{Contabilidad y modos de producción}

La contabilidad es una construcción socio-histórica, una creación humana. En esta vía, es importante mencionar que al ritmo cultural en que la forma de capturar la riqueza cambia, la contabilidad evoluciona y se moldea de acuerdo con las necesidades y características socioeconómicas del contexto, así que cada cambio en la realidad (cada cambio en el paradigma dominante), es decir, cada cambio en los modos de producción, y las relaciones entre el capital, la tierra y el trabajo, determinan cambios en la contabilidad, pero no de forma gratuita, sino interesada, para representar, controlar y legitimar el sistema social dominante.

Modo de producción es el gran concepto teórico que expresa el proceso histórico de producción y creación de bienes e ideas. Se trata de una unidad de conjunto que involucra variables económicas, políticas e ideológicas, en una compleja trama de relaciones y articulaciones que demarcan su ámbito de influencia. (Suárez, 2004, p. 17)

La teoría marxista indica que los modos de producción, las formas de intercambio y la división del trabajo determinan la estructura de mando de la sociedad como relaciones históricas y productivas para ubicar el curso de las naciones. "Un modo de producción es al mismo tiempo una forma específica de la organización de las relaciones sociales de producción, junto a las correspondientes relaciones de distribución, y un modo de dominación social organizado institucional y políticamente" (Chesnais, 2016, p. 5). Estas formas de organización no actúan nominalmente, sino que co-construyen dispositivos de medición y valoración de sus movimientos, elementos que no sólo son de orden contable, pero encuentran en la contabilidad una base teórica, tecnológica y técnica para representar metódicamente la realidad y articular las fuerzas de producción a un sistema de medición que le permita alcanzar objetivos sociales y políticos claros.

En este orden de ideas, los modos de producción además de ser una forma de entender la organización de las sociedades en torno a los factores económicos de producción permiten argüir que cada periodo histórico articulado a un modo de producción contrae consigo un objetivo distinto, objetivo del cual la contabilidad da cuenta moldeando su conocimiento y sus lecturas de la realidad a través de las formas de apropiación de la riqueza para representarlo.

"La Contabilidad no apareció como un fenómeno azaroso, sino específicamente como respuesta a una necesidad general y mundial" (Hatfield, 1924 , p. 269). Las necesidades humanas provienen de todas las esferas del entendimiento, no obstante, las más recurrentes y expresadas son necesidades materiales de las cuales la contabilidad da cuenta. A continuación, se presenta una gráfica que resume perspectivas históricas de la contabilidad de acuerdo 
Arias, J.; Cano, V. Contabilidad y modos de producción...

con la visión clásica de la historia y con la articulación de los modos de producción.

Gráfica 1. Evolución histórica de la contabilidad

Edad Antigua:

(4000 a.C. -475)

Caída del Imperio Romano de Occidente por los

bárbaros

Edad Media:

(476 - 1453)

Caída de Contantinopla en poder de los otomanos

Edad Moderna:

(1453 - 1789)

Revolución Francesa

Edad Contempóranea:

(1789 hasta nuestros días)

Llegada del hombre a la Luna
Modo de producción esclavista

Invención de la escritura y los números. Creación de unidades económicas con base en la división del trabajo.

Modo de producción feudal

Contabilidad por partida simple y de tipo memorial, aparición del crédito y de sociedades comerciales, representación comercial por contratos de comisión.

\section{Modo de producción mercantilista}

Generalización del uso de la imprenta; publicación de la Summa de Paciolo; contabilidad por partida doble; nace el capitalismo en su fase mercantilista; y surgimiento del gremio de contadores.

Modo de producción capitalista

Revolución industrial; expansión del comercio; desarrollo de la contabilidad de costos; fases industrial, financiera e imperialista del capitalismo; desarrollo de nuevos modelos contables.

Fuente: tomado de Suárez (2008).

Es importante precisar que el transcurrir histórico no es lineal ni se puede contar como un relato ordenado y coherente. Los sucesos históricos son diversos, relacionales e influyentes en cada latitud, de allí que varios modos de producción pueden compartir el mismo periodo histórico en diferentes zonas geográficas; la contabilidad se manifiesta en todas las configuraciones de los modos de producción y responde a objetivos distintos dependiendo del contexto, lo cual impacta la perspectiva de estandarización de las normas de información financiera, pues siguiendo los desarrollos de los modos de producción, el papel y sentido de la contabilidad no puede ser el mismo en todo el mundo desde que los factores de producción sean distintos.

De esta forma, en el siguiente apartado se presentará algunas aproximaciones al papel social de la contabilidad en los modos de producción: comunitarismo primitivo, esclavismo, feudalismo, capitalismo mercantil, capitalismo industrial, capitalismo financiero, capitalismo bursátil, socialismo y comunismo. 


\section{III.1 La contabilidad en el comunistarismo primitivo}

El origen de la contabilidad es catalogado como incierto desde una concepción disciplinar amplia. Desde los rasgos más remotos conocidos por los humanos coexisten debates sobre la pertinencia, suficiencia y validez de las pruebas históricas, antropológicas y arqueológicas para identificar las manifestaciones humanas. Cuando se trata de identificar el papel social de la contabilidad en el modo de producción conocido como comunitarismo primitivo, periodo histórico enfocado a la utilización de la fuerza de trabajo para desarrollar actividades como la caza y la recolección de alimentos, el raciocinio humano generó formas primigenias de clasificación y de control. Los estudios de Sy y Tinker (2006) muestran que el origen de la contabilidad podría estar en las prácticas de seriación en huesos de babuino, hechas por los nativos de Ishango (actual República Democrática del Congo), las cuales han sido datadas a través de pruebas de carbono 14 , y pueden ostentar más de 18.000 años de antigüiedad.

Algunos historiadores, geólogos y arqueólogos ortodoxos consideran que estos huesos sólo son sumatorias, sin embargo, existe la perspectiva de que son y representan formas de control de elementos. Claramente la asociación que se le da a este hecho con la contabilidad es bajo la noción de racionalidad controladora explicada en apartados anteriores, ya que la función primaria de la contabilidad es esa, así sus actuales técnicas y métricas sean múltiples y matematizadas. Los instrumentos rudimentarios de los humanos en este modo de producción fueron muy simples, pero necesitaban un control y organización, que en esencia se daba a través de su racionalidad de saber qué se posee, característica natural de los seres humanos. Además, la profesora Denisse Schmandt-Besserat, junto a Richard Mattesich, realizaron un trabajo arqueológico en Irak, donde hallaron fichas contables cercanas a los años 8000 a.C. La investigación denota que son objetos de cálculo abstracto y control de recursos de personas de la antigua Mesopotamia. El papel social de la contabilidad en este modo de producción se enmarca en la percepción de control de la riqueza existente, en un sentido genuino dadas las no identificables características de acumulación o ganancia material de la época. Su lógica temporal de control se enfoca en el presente y la utilidad práctica se enmarca en apoyar actividades cotidianas.

\section{III.2 La contabilidad en el esclavismo}

El esclavismo se configuró como un modo de producción con una significancia de la noción de 'propiedad privada', siendo ésta muy importante para entender el papel de la contabilidad en la idea de dar cuenta de la realidad de un propietario, es decir, asignándole a un ser la tenencia de bienes o deudas. En un sentido general, cuando las comunidades se asentaron en un 
espacio geográfico específico, dejando progresivamente su estilo de vida como nómadas, se apropiaron de una porción de tierra, de los recursos naturales que allí se disponen y, más aún, de sus cosechas para con ello incentivar dinámicas de intercambio; en este proceso de asentamiento, muchos individuos se vieron desposeídos de tierra, sin más recurso que su fuerza de trabajo, que no fue insertada de manera natural en un sentido de oferta y demanda en las bases estructurales del naciente comercio, sino que fue sometida y privatizada por ejercicios de poder que justificaban una supremacía desde diferentes perspectivas, en algunos casos por determinantes económicos, étnicos, raciales o religiosos, a razón de la propiedad y escasez.

En este modo de producción, los humanos ejercen poder sobre la tierra, los recursos naturales y otros humanos (desposeídos de tierra), poder que fue legitimado desde las nacientes instituciones políticas, sociales, eclesiásticas y económicas. Allí, las principales formas de control de los recursos y la riqueza fueron efectuadas mediante anotaciones e inscripciones en superficies que sirvieran para tal fin, desde recursos de la tierra (como piedras, tallos de árboles, arcilla) e incluso en las paredes o cimientos de las construcciones y lugares donde se llevaban a cabo ejercicios de intercambio, tal y como los templos o palacios.

\section{3 La contabilidad en el feudalismo}

En este modo de producción, la contabilidad tiene un aspecto más registral y se va consolidando el intercambio en especie (trueque), luego con monedas de oro y finalmente con el papel-moneda. El feudalismo es un modo de producción basado en la tenencia y explotación de la tierra (no como terreno o territorio, sino como espacio geográfico apto para la producción, primariamente agrícola). En esta forma de articulación de los factores de producción la noción de acumulación es importante y la contabilidad se preocupa por ello. En esta etapa la contabilidad es fundamentalmente de partida simple (conteo básico a modo de inventario), aunque se desarrollan conceptos que van a ser muy importantes para la partida doble en cuanto el mercantilismo entre en auge.

En el feudalismo el dueño de la tierra cumple un papel importante desde la lógica de la propiedad material y el dar cuenta de la riqueza de la hacienda. El papel social de la contabilidad cambia porque las formas de la escritura y la numeración se hacen presentes para generar partidas de registro básico controlando las existencias de las comunidades y el Señor Feudal a partir de lo producido por los siervos; la contabilidad allí se enmarca dentro de un sistema de administración del poder al ser garante de los movimientos de los recursos.

\section{III.4 La contabilidad en el capitalismo mercantil}

En esta etapa, la contabilidad toma proporciones sociales de mayor envergadura, ya que en lo mercantil los intercambios de bienes se afianzan, la 
noción de dinero y propiedad privada son preponderantes para las relaciones humanas. Las formas de acumulación a partir del intercambio se intensifican y la riqueza económica es tomada como el núcleo de desarrollo. Se continúa con la gestación de una partida doble (relación entre costos de adquisición, inventarios y utilidades), lo cual configura otras lógicas de acumulación; este cambio se da en Italia, con Benedetto Cottrugli y Luca Pacioli, quienes hacen una contabilidad "a la veneciana" en el siglo XVI, para dar control de las fluctuaciones del capital, fundamentalmente por operaciones navales. Es notorio que en este momento histórico la contabilidad es controlada por los dueños del capital y se empieza a consolidar un mayor interés por saber qué se posee materialmente, lo cual también establece la consolidación de la propiedad privada y el vínculo de la contabilidad para legitimarla.

\section{III.5 La contabilidad en el capitalismo industrial}

El viraje de los factores económicos de producción hacia el capital en lógica acumulativa, la revolución industrial y el despliegue mundial por la producción a escala, genera cambios en la vida social. La contabilidad, ya consolidada como partida doble reconociendo activos, pasivos y patrimonio, centraría su mirada en la maximización de utilidades y minimización de costos de producción. Este periodo histórico representó cambios estructurales en las formas de medición y valoración de la contabilidad; la economía de los servicios aún no era representativa, sino la fabricación de productos. Allí la contabilidad configura un sinnúmero de metodologías y técnicas de medición, valoración y control del capital, sus costos y sus actores implicados, y se consolida un nivel de racionalización de la contabilidad, donde todo debe estar controlado y registrado "fielmente" para las necesidades de las unidades productivas. Las valoraciones desde la contabilidad empiezan a determinar el rumbo de los negocios, los salarios de los obreros (a través del cálculo de rentabilidad entre precio de venta y costos), y a dar cuenta de los intercambios del capital.

El papel social de la contabilidad estuvo enfocado en la medición de la productividad industrial, es decir, la contabilidad subyuga su conocimiento al capitalismo económico y del valor, la presupuestación de costos y gastos se enmarca como la actividad más relevante, y las formas de medición se pueden observar al estilo foucaultiano de 'vigilar y castigar', la contabilidad proporciona el rendimiento necesario para generar la utilidad esperada como un dato o métrica que se debe cumplir. La noción privada de organización se consolida y la contabilidad se erige como instrumento al servicio del cálculo utilitario.

\section{6 La contabilidad en el capitalismo financiero}

En esta etapa, fundamentalmente en lo recorrido del siglo XXI, la contabilidad sufre un cambio histórico, pues la preponderancia de 
representación no son los hechos económicos (visión histórica), sino los pronósticos financieros (visión futura), debido a los cambios en las relaciones del capital. Es decir, la contabilidad empieza a pasar de controlar y legitimar las actuaciones de los industriales burgueses, a controlar y legitimar las fluctuaciones del capital financiero a través de las bolsas de valores y las acciones empresariales de corte proyectivo de los capitalistas financieros especulativos. Es claro que la contabilidad, debido a su racionalización y particularización de la realidad, desmenuza los hechos que se generan alrededor del capital, y éstos actualmente dan cuenta de que no interesa tanto que tan eficiente sea una organización o el sistema, sino que tan rentable lo sea, es decir qué tantos flujos futuros de efectivo pueda generar. La idea de que el dinero genera más dinero, sin necesidad de mediar una relación productiva, sino sólo con la consolidación de la tasa de interés, representada fundamentalmente en la actividad bancaria, consolida otra lógica de acumulación que debe ser captada por la representación contable.

Lo anterior son las formas actuales de dominación del capital, sólo valores de cambio y rentabilidad desde la visión más utilitarista de la economía neoclásica. El papel de la contabilidad es probabilístico, con cálculos del valor del dinero en el tiempo, con tasaciones de intereses donde el pasivo de las organizaciones tiene mayor representatividad y la razonabilidad económica de los estados financieros toma más importancia que el cálculo fiscal.

\section{III.7 La contabilidad en el capitalismo bursátil}

El modo de producción denominado como capitalismo bursátil es una mutación del capitalismo financiero presente en el siglo XXI. Su lógica está basada en la creación de riqueza a partir de operaciones con variables diversas, sin probabilidades garantizadas, es decir, variables inciertas. Basado en la teoría de juegos, la econometría, la estadística avanzada, el riesgo financiero, este modo de producción se basa en la gestión del capital material e inmaterial, pero de orden especulativo, con creación de tendencias y expectativas que no siempre tienen contraste fáctico. En el entorno contable, tradicionalmente el costo histórico con base en el comercio y la construcción de valor económico daban sentido a las transacciones; ahora ni siquiera las proyecciones probabilísticas con bases razonables ejercen dominio. Es la variable especulativa e incierta, de la maximización constante del posible valor financiero de un objeto, marca o una acción la que genera cambios vertiginosos e inciertos en la aparente realidad monetaria.

En este periodo el presupuesto es una herramienta cuasi-obsoleta, ya que no hay base razonable para anticiparse a los hechos, ni crecimientos progresivos. Esto no implica que no se pueda crear un imaginario social con escenarios futuros, sino que los cambios vertiginosos, no provenientes de la articulación 
de fuerzas productivas que creen valor, están más atados a fenómenos psicopolíticos en mercados imparciales, inciertos e interesados, que al factum económico.

El papel de la contabilidad en este modo de producción muta hacia la representación de lo que no siempre tiene soporte tangible. "Para decirlo en forma breve, es la dictadura de la Bolsa sobre los fines de las empresas" (Aglietta y Berrebi, 2007, p. 27), debido al desmantelamiento de las fuerzas productivas para la consolidación de las fuerzas crematísticas fluctuantes.

\section{III.8 La contabilidad en el socialismo/comunismo}

A pesar de que sociológicamente y políticamente no se puede equiparar el modo de producción socialista con el comunista, debido a sus etapas en la transformación social, a sus concepciones teóricas entre "equidad", "igualdad", "socialización" y "grado de regulación estatal", y a los casos prácticos de implementación de estas formas de articulación económica en la realidad mundial: Unión de Repúblicas Socialistas Soviéticas, Alemania, Cuba, Corea del Norte, sólo por mencionar países con cambios más representativos, sí se pueden aglutinar dentro de una categoría donde los factores de producción contemporáneos no están centrados en el capital, ya que no se trata de ver la participación puntual de la contabilidad en cada país no-capitalista, sino de encontrar las generalidades de la contabilidad en un modo de producción no alineado con el capital.

Estos modos de producción centran su atención en mitigar los desequilibrios económicos de la distribución de la riqueza y de fortalecer las instituciones estatales reduciendo libertades de maximización de acumulaciones, pero garantizando condiciones dignas de vida humana. En estos momentos históricos "la contabilidad y el control se transforman en un medio de defensa de los intereses de toda la sociedad, sirviendo a los objetivos de todos sus miembros" (Sánchez, 2003, p. 198). De hecho, "los países socialistas desarrollan en primer lugar los modelos y técnicas de contabilidad social o nacional, los cuales delimitan gran parte del quehacer contable de la empresa, quedando la contabilidad empresarial en lugar secundario y con una estructura muy esquemática" (Bueno, 1972, p. 247), lo cual desafía las formas e interpretación de los procesos de medición, valoración, control y representación de la contabilidad.

La contabilidad en la sociedad socialista es un arma poderosa del control continuo e interrumpido de los procesos de cumplimiento de los planes y la movilización de las reservas productivas en las distintas organizaciones y ramas de la economía nacional. De esa manera dicha contabilidad cumple con las funciones siguientes: 1) La creación de la información necesaria para el control sobre el registro de las transacciones efectuadas, sobre el cumplimiento del plan. 2) La dirección de la actividad en las 
Arias, J.; Cano, V. Contabilidad y modos de producción...

organizaciones. 3) La activa influencia sobre la eficiencia del trabajo. 4) El control y conservación de la propiedad social sobre los medios de producción. (Sánchez, 2003, p. 199)

"En todas partes tiene lugar un sabotaje sistemático, inflexible, de todo control, de toda vigilancia y de toda contabilidad" (Lenin, 1962, p. 125), lo cual termina enriqueciendo o empobreciendo poblaciones. Esta perspectiva de Lenin hace entender la importancia y relevancia que se le da a la contabilidad, pues el mismo Vladimir Lenin resaltó el papel de la contabilidad en la consolidación del socialismo, argumentando que en el proceso de transición del capitalismo al socialismo, "la organización de la contabilidad y el control pasa a ser el centro de gravedad (...); sólo por este medio puede prepararse la feliz terminación de la lucha contra la burguesía, es decir, el afianzamiento total del socialismo" (1962, p. 372).

Conforme con lo anterior, la contabilidad puede actuar en diferentes sistemas de desarrollo económico, que no necesariamente tienen que ser el socialismo o el comunismo, sino en cualquier esfera social que se piense dinámica de intercambio de capital diferente, siempre y cuando escapen a la lógica capitalista y cumplan con factores de actuación distintos. Ello implica ubicar una contabilidad con objetivos claros dentro de estos modos de producción, fundamentados en el control de la existencia con fines más tributarios que de especulación financiera, como acumulado histórico, y como instrumento clave para determinar quiénes y en qué medida sobrepasan positiva o negativamente las brechas de equidad de la sociedad. En este contexto, la contabilidad minimizaría su preponderancia en las cuentas producto de la financiarización para tomar mayor atención a las patrimoniales y de control de bienes para el reporte estatal.

\section{Consideraciones finales}

El papel social de la contabilidad en cada modo de producción es disímil, sin embargo, conserva tres elementos de su esencia como saber: la racionalidad controladora, la representación de la realidad y su participación en el accountability sociopolítico. Esta tríada de elementos es concomitante en el transcurrir sociohistórico; los puntos de diferenciación donde se encuentra la participación social de la contabilidad están presentes en los objetivos políticos de la comunidad y en las particularidades de los fenómenos derivados de la dinámica societal. La contabilidad responde al contexto económico y social, y transforma su praxis de acuerdo con las presiones exógenas, es decir, es una disciplina en constante transformación práctica. Sus usos y desusos pueden ser tan aportativos como destructivos para el desarrollo ambiental y humano.

La contabilidad ha actuado como "base racional" para la toma de decisiones en tiempos de certidumbres e incertidumbres económicas y como 
una "representación razonable" en un contexto financiero especulativo, acoplando sus andamiajes téorico-prácticos para reconocer, medir, valorar, consolidar, controlar, revelar y representar múltiples realidades. Además, se encuentran elementos que se escapan a la reproducción del capital, pero que desafortunadamente son subyugados en tecnicidad y teorización por la lógica financiera imperante. Se resalta la intimidad de la contabilidad con las decisiones políticas y económicas en cada modo de producción, y a su vez, su papel de legitimación de las acciones regentes del contexto. No obstante, es claro que la unificación de los criterios y las normas de la contabilidad no proceden tangencialmente, pues si la contabilidad históricamente se ha adecuado a las condiciones culturales, con herramientas y representaciones distintas en cada modo de producción, hasta la fecha no existe ninguna norma que logre abarcar realidades diversas, complejas e inciertas y capturarlas en un enunciado incluyente y coherente con la realidad llamado estándar.

Ya lo dirían Fearnley y Sunder al problematizar la estandarización contable: "no two transactions or events are identical (...) Varying interpretations of transactions in different countries create additional problems" (2006, p. 26). Si se entiende que cada modo de producción genera un contexto e interpretación diversas de la realidad, no hay espacio para pensar en una contabilidad unificada mientras haya preponderancia de algún factor económico, salvo que las condiciones sociales, políticas, económicas, culturales e incluso ambientales y sus relaciones sean análogas a escala global (aunque eso no las hace comparables dada la complejidad humana y las redes interpretativas); lo diferente a una contabilidad poli-valorativa de acuerdo con su contexto son ópticas miopes, interesadas y con sesgos para la comprensión económicoproductiva y social de las naciones.

\section{Referencias bibliográficas}

Aglietta, M. y Berrebi, L. (2007). Désordres dans le capitalisme mundial. París: Odile Jacob. Arias, J. D. (2014). El papel de la contabilidad en sistemas de desarrollo alternativos. Adversia, 15.

Arias, J. D. (2017). Ecología Política: Desafíos de la Contabilidad frente a la Justicia Ambiental. En-Contexto, 5(6), 303-326.

Arias, J. D. y Cano, V. (2018). Análisis de la perspectiva transdisciplinar como estrategia pedagógica de enseñanza y de aprendizaje en Contaduría Pública. En: [C. M., Pico - Comp.). Experiencias de aprendizaje significativo para la apropiación de conocimientos en ciencias económicas, administrativas y contables. Bogotá: Editorial Politécnico Grancolombiano. ISBN: 978-958-8721-89-7

Ariza, E. D. (2000). Las relaciones de producción y la partida doble. Legis del Contador, 2, 97-132.

Belkaoui, A. R. (1993). Accouting Theory. 3a. ed. Chicago: The Dryden Press. 
Arias, J.; Cano, V. Contabilidad y modos de producción...

Bueno, E. (1972). La Planificación de las Empresas Socialistas: aspectos financieros y contables. Revista Española de Financiación y Contabilidad, 1(3), 661-692.

Cardona, J. D. y Hinestroza, Y. (2013). La planeación y el presupuesto como sistemas simbólicos articulados a los procesos de resistencia campesina: análisis de una organización a partir de los referentes teóricos de Pierre Bourdieu. Activos, 20, 55101.

Chesnais, F. (2016). El curso actual del capitalismo y las perspectivas para la sociedad humana civilizada. Revista o olho da história, 24. Disponible en: http://oolhodahistoria. ufba.br/wp-content/uploads/2016/12/francois.pdf

Chua, W. F. (1986). Radical Developments in Accounting Thought. The Accounting Review, 61(4), 601-632.

Fearnley, S. y Sunder, S. (2006). Global reporting standards: the Esperanto of accounting. Accountancy, 137.

Gil, J. M. (2003). Conocimiento contable e identidad latinoamericana. Porik An, 7-8(5), 17-77.

Hatfield, H. R. (1924). Defensa histórica de la contabilidad. The Journal of Accountancy, 37(4), 241-253.

Lenin, V. (1962). Obras Escogidas Tomo II. Moscú: Editorial Progreso.

Quinche, F. L. (2006). Historia de la Contabilidad: Una revisión de las Perspectivas Tradicionales y Críticas de Historiografía Contable. Revista Facultad de Ciencias Económicas: Investigación y Reflexión, XIV(1), 187-201.

Sánchez, L. M. (2003). Propiedad, planificación y control. Elementos indispensables de la gestión económica en el socialismo. Revista Santiago, 103, 191-206.

Sarmiento, H. J. (2007). Retorno a la Idea. Elementos metodológicos para la construcción de conceptos propedéuticos de investigación contable. Lúmina, 8, 43-57.

Suárez, J. A. (2004). Arqueología e historia de la contabilidad. En: Cosmovisión histórica y prospectiva de la contabilidad. 1a ed. Bogotá: Universidad INCCA.

Suárez, J. A. (2008). Momentos estelares de la contabilidad. Bogotá. Universidad La Gran Colombia.

Sy, A. y Tinker, T. (2006). Bury Pacioli in Africa: a bookkeeper's reification o accountancy. Abacus, 42(1), 105-127.

Weber, M. (2010). La Ética Protestante y el Espíritu del Capitalismo. Bogotá: Libros Hidalgo. 